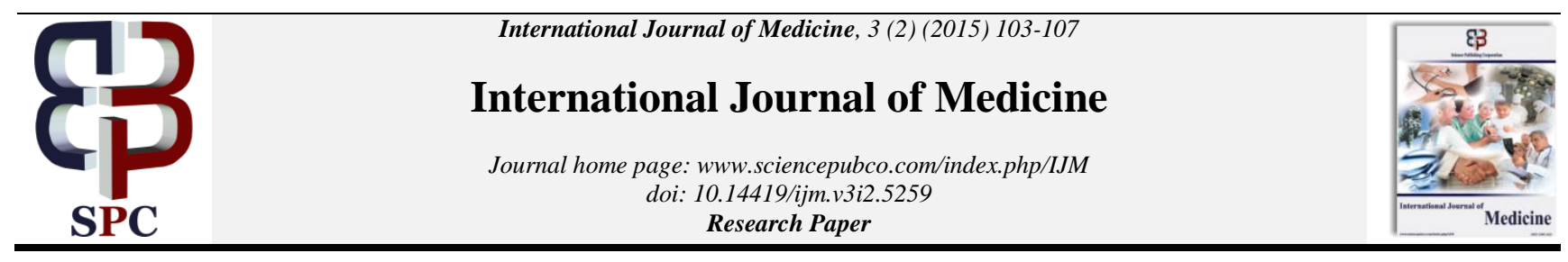

\title{
Evaluation of the reflective practice programme
}

\author{
Lucia N. Nelumbu \\ University of Namibia, Private Bag 13301, Windhoek; Namibia \\ E-mail: lnelumbu@unam.na
}

\begin{abstract}
It was obvious that the developed programme would be evaluated after the implementation process. The programme was evaluated after four months of implementation on the application of the content of the programme. The researcher organised a focus group discussion for the registered nurses in order to gain information regarding the implementation of the developed programme. The participants were invited on basis of their participation in the previous implementation and training workshop of the reflective practice programme. The participants concluded that this is an effective and fruitful approach for all health providers, which is helping them to identify their mistakes and to reflect on their daily practice in order to make improvements. This approach assists them on how to set priorities and assign tasks to other colleagues to work as a team. Moreover this programme helped the registered nurses to know how to assist the patients even when they did not call for help and also to realize the need to attend to the patients' calls immediately. This programme also drew the attention of the registered nurses to the necessity of treating patients as human beings.
\end{abstract}

Keywords: Evaluation; Reflective Practice; Focus Group.

\section{Introduction}

Evaluation is the process of collecting and interpreting data to determine the accomplishments, strengths and weaknesses of the programme. It also intended to ascertain how the registered nurses viewed the application of the programme to their clinical practice. The evaluation focused on assessing the extent to which the programme objectives have been met. The evaluation furthermore sought to determine whether the training of registered nurses was effective and whether the programme is acceptable to the registered nurses. The purpose of discussions after the implementation was to evaluate the effectiveness and efficiency of the developed programme. The above purposes should be viewed in the light of arguments made by different authors who described programme evaluation as the effort to determine whether programme objectives have been reached and to record the gathering of information to assess the efficiency of a programme (Musal, Taskiran, Gursel, Ozan, Timbil, \& Velipasaoglu, 2008).

\section{Purpose of evaluation}

The purpose of evaluation was to determine whether the developed programme has succeeded or not in the facilitation of reflective practice during the daily practice of registered nurses. It also intended to ascertain whether reflection by practitioners has improved during implementation of an educational programme of reflective practice to determine the effectiveness of the programme.

\section{Evaluation process}

The evaluation of the programme was carried out 18 weeks (about four months) after its implementation. The registered nurses were given a period of twelve weeks within which they should implement the programme before it was evaluated.

After twelve weeks (three months) the participants were invited to attend a half-day workshop in order to evaluate effectiveness of the developed programme. Due to some limitations, the initial workshop for evaluation did not materialised as it was planned. Consequently, the evaluation was rescheduled and took place after four months, where it commenced with a focus group discussion which attended by five participants.

The follow-up evaluation was participated, in by a total of nine registered nurses out of fifteen (15) who attended the training workshop. The initial number could not be retained due to the unforeseen circumstances beyond the researcher's control. Not all nine registered nurses participated in the focus group discussion. However, the researcher managed to bring together five (5) registered nurses who attended the initial focus group discussion. The focus group discussion lasted about 90 minutes. This was succeeded by individual interviews with four registered nurses that were conducted a week later. It was not easy to bring the participants together. The researcher who also served as the moderator had to obtain the verbal/telephonic consent through their supervisors in their respective units/wards for the participants to attend the evaluation process.

In spite of the hindrances described above, the evaluation process was conducted. The venue for the focus group discussion was comfortable and conducive to discussions and free from outside distractions and noise. The researcher, who acted as moderator requested two colleagues to serve as co-moderators. All the participants, the moderator and co-moderators sat around a table which enabled them to see and hear one another well.

The moderator (researcher) welcomed the participants to the focus group discussion, after which she introduced herself and the comoderators and also asked the participants to introduce themselves. The moderator (researcher) gave a brief overview of the 
topic to be discussed including the purpose of the evaluation, as well as the intended duration of the discussion. The moderator explained how the discussion was to be recorded-for example, the use of note taking and tape-recording. Permission for taperecording was sought from the participants and granted. The researcher explained further to the participants that all contributions or views were valued and would be kept confidential and anonymous. The researcher further assured the participants that they were regarded as knowledgeable and experienced on certain nursing situations and patient needs about which she and comoderators hoped to learn and therefore would value the participants' opinions. The researcher further indicated that it was important for them to hear every participant's ideas and opinions. She also pointed out that there were no right or wrong answers to questions. All ideas, experiences and opinions were valuable.

\section{Method of evaluation}

The evaluation was done through the focus group discussion method. A focus group discussion is a method of discussion designed for small groups. The focus group is described as a group which include participants with a shared experience or concern identified in advance by a researcher (Forneris \& PedenMcAlpine, 2006). Again, according to the literature's description, a focus group can be of particular assistance in identifying factors of importance (Bengtsson \& Ohlsson, 2010). Although Bengtsson and Ohlsson (2010) clarified that a disadvantage of the focus group is that some participants may feel uncomfortable to express themselves in front of a group of people, in this study, however, all participants in the group were already familiar with each other. The focus group is a method of group discussion in which the interaction between the moderator and the group, as well as the interaction between group members, serves to elicit information and insights in response to carefully designed questions.

Focus group discussion was described as a research methodology in which a small group of participants gather to discuss a specified topic or an issue to generate data. The main characteristic of a focus group is the integration between the moderator and the group, as well as the interaction between group members. It was further explained that instead of just the moderator asking questions, the group members are encouraged to communicate with one another, exchanging ideas and comments on each other's experiences or points of view. The purpose of the focus group discussion was to gain knowledge and learn perspectives of participants on the implementation of reflective practice programme (Wong, 2008).

To facilitate the focus group discussion, the researcher developed a guide consisting of questions on the effectiveness and applicability of the developed programme. The purpose of the discussion guide in a focus group discussion was to provide a framework for the moderator to ask probing questions (Wong, 2008). The probing questions in the discussion were designed to stimulate further discussion. The researcher acted as the facilitator and explained the purpose of the interview and how the focus group could contribute to the data collection. The researcher used the coding statements of individual participants in order to investigate similarities and differences. Each participant was allocated a number in order for the moderator to be able to compare the participants' statements. The focus group employed two co-moderators who took careful notes of the participants' statements.

The focus group discussion was guided by open-ended questions. Key questions were carefully guided to get to the point of the desired information. The technique used to collect information from the focus group discussion was a combination of note taking and an audio tape recorder to capture essential information.

The researcher (moderator) served as the leader of the discussion during the focus group discussion. In leading the discussion the researcher kept the conversation flowing. She took some notes of the comments made by the participants to use as references, and also operated the tape recorder at the same time. The researcher (moderator) constantly followed the facilitation guide she had developed beforehand. The moderator's role was also to look after the group dynamics and made sure that all participants joined in the discussions. The researcher (moderator) used probes such as:

- Would you explain further?

- Can you please describe what you mean?

- Did anyone have a different experience?

The moderator and the co-moderators captured and recorded the important information that was expressed by the participants.

All participants had the opportunity to discuss their experiences of learning in practice. Careful facilitation was maintained in order to ensure that individual participants did not dominate the group. During the focus group discussion the moderator demonstrated the listening skills. This was important for the participants to know that the researcher (moderator) paid attention to what they were sharing. This approach allowed the researcher to probe effectively at the appropriate points during the discussion.

The participants' responses were discussed. The focus group discussion proceeded until it reached saturation level where there was repetition of views and no new information was shared. After the end of the focus group discussion a short summary of the discussion was presented and the participants were asked to indicate if the summary was an accurate representation of their views. The participants were asked to summarise their positions and to look for any information which might have been omitted.

During the focus group discussion an audio-tape was also used with the participants' permission. The researcher controlled the tape recording, monitored the time periodically to make sure that the discussion progressed appropriately, and ensured that all registered nurses who participated took part in the discussion. The audio-recorded discussion and interviews were later transcribed and analysed. The moderator thanked the participants for their time and participation and assured them that the information they had shared was valuable.

\section{Data analysis}

Analysis of the focus group data was carried out. Transcribing of data was done and compared with the handwritten notes taken by the co-moderators and the moderator to fill in all the gaps. This involved sorting out the information and assigning it according to categories. Coding was followed according to the numbers allocated to each participant in order to categorise the information easily. The researcher (moderator) decided to use the interpretive level of analysis whereby she created connections between perspectives.

\section{Discussions of findings}

Participants were requested to highlight their experiences of the reflective practice programme, elaborating on the positive and negative aspects of the developed programme. They were also afforded the opportunity to raise questions and to make recommendations. Exploring the introduction of reflective practice to registered nurses in the training hospitals, the researcher observed that the registered nurses perceived it as a new approach to which they needed to adapt, as it was different from their prior educational and practical experience.

The evaluation was guided by the following main question which was accompanied by probing questions to elicit responses:

Main question

How did reflective practice programme assist you in improving your daily practice?

On the above question the respondents replied as follows:

- "It assisted me in setting priorities and assigning tasks to other colleagues."

- "It helped me to bring improvement to my colleagues in keeping proper records." 
- "I have managed to look at things in a positive way even in a critical moment."

- "I learnt to teach others to reflect back and use knowledge they have acquired during their clinical practice."

- "It gave me the direction to look at the patients holistically, for example when doing the audit I have to see whether the report has been written in a problem-oriented way, according to the diagnosis or problem the patient has been admitted with."

- "It also has helped me to guide/encourage the ward staff on the importance of proper record keeping and how they can improve."

- "Reflective practice updated my knowledge and made me realise my shortcomings."

- "It taught me that patients change daily and I need the application of reflective practice to manage it."

- "Firstly I would like to say that my colleagues in OPD (registered nurses) even after the feedback was given to them felt that this programme is concerned with the registered nurses in the clinical wards only and not necessarily the outpatients 'staff, so they responded negatively to it. Though on a daily basis they are practicing it, as it is heard in their daily patient problem discussion they encounter and the approach they decided to take as well as the evaluation and action taken toward this."

- "One needs to further discuss and educate them on this and emphasise its importance though they do it unknowingly it should be acknowledged, learnt and put in general practice for all registered nurses."

- "It helps me to reflect back on my daily practice/ action."

- "With the reflection or thinking back to what you did will help you to improve what you did or to put more effort on what you are doing to get the solution to the problem."

- "It helped me to make the correct decision/option."

- "It will equip you with knowledge and skills regarding practice."

- "It was an eye opener for me."

- "It is kind of a scientific method."

- "It is useful especially in nursing process."

- "We learnt something that will help us in identifying our mistakes and make the improvement."

- "I am not involved in actual daily practice. This is a process needed for us to practice professionally."

- "People really do not know what reflection is all about they just practice a routine, no reflection."

- "I made a plan of action-a programme to teach the staff whereby we started with assessment stage, reflecting back on previous knowledge and plan what action we will take. People do not attend "in-service training", either registered nurses or enrolled nurses. This is a challenge."

- "We had a meeting after the workshop to discuss on how we can implement this into our nursing practice. It seems that we need to do it in a scientific way. We have to ask ourselves on 'what went wrong'. We learnt something from this that we can apply to our practice. We plan to have official meeting with our staff in order to discuss this."

- "It helped me on how to treat a person as a human being. To attend immediately to the patients' calls."

- "I learnt on how to tackle my patient's needs."

- "I got to know how to help my patients even if they do not ask for it I know what to do."

- "This is effective and fruitful approach."

- "I am now able to reassess my patients."

Summary of findings on question one

On question one nearly all the respondents indicated that the reflective practice programme was an eye-opener for them. They saw it as a kind of scientific method which is useful especially in the existing nursing process. They concluded that this is an effective and fruitful approach for all health providers, which is helping them to identify their mistakes and to reflect on their daily practice in order to make improvements. This approach assists them on how to set priorities and assign tasks to other colleagues to work as a team. Moreover this programme helped the registered nurses to know how to assist the patients even when they did not call for help and also to realize the need to attend to the patients' calls immediately. This programme also drew the attention of the registered nurses to the necessity of treating patients as human beings. It was also pointed out that the reflective practice programme assisted the registered nurses to guide and encourage the ward staff to realize the importance of proper record keeping and how they can improve. The participants further indicated that they learned to teach others to reflect back and use knowledge they have acquired during their clinical practice.

In the second step, the following question was asked.

What did you like most about the reflective practice programme?

On this question, the following are the responses from the respondents:

- "What I like from this process is to review my work where I can do changes and teach others to improve."

- "The fact that you look back."

- "Reflective practice is a reminder to look back to the client/patient's condition."

- "It is a very good programme to keep the nurses on alert. We can make the diagnosis correctly and handle situations. Situations that reminded me what to do and how I effectively managed it."

- "It is very beneficial on health situations."

- "Reflective practice helped me / reinforced me to do something correctly."

- "I like reflection because this is showing me what I should do."

- "During in-service training I have to make sure that nurses understand and learn something from me. If they do not understand I have to see what I can do so that they can understand better."

- "I like the way that this will benefit the nursing profession if we will apply it in our daily practice."

- "We want to start with reflective practice in our daily practice, because this is even important to apply at our homes in our family."

- "It helped my colleagues to provide spot teaching on clients."

- "I like Kolb's experiential learning and Rolfe's reflective framework and I want to apply into practice."

- "Reflective practice programme gave me positive thoughts."

- "I got to think back on how to nurse the patient and how to reflect back."

- "I like the revision of assessment, planning and evaluation."

Summary of findings on the above question or item

The responses here indicated that they like the reflective practice programme because it gave them some positive thoughts on how to nurse the patient and reflect back. The registered nurses pointed out that Kolb's experiential learning and Rolfe's reflective practice framework helped them to understand how to apply reflective practice into practice. It was concluded that reflective practice will benefit the nursing profession if the health providers will apply it in their daily practice. Some of the registered nurses expressed that what they like about this programme is the fact that it a very good approach that keeps the nurses alert and reminds them to look back to the patients' conditions as well as to review their work and see where they can improve or where they need to teach others to improve.

In the third step the following question was asked:

What do you believe should be changed and why do you think it is necessary?

In answering the above question the respondents stated as follows:

- "Reflective practice is very important and informative."

- "Many registered nurses should attend. All nurses need the information." 
- "Enrolled nurses should also be taught on how to apply reflective practice in order to improve quality care for the patients."

- "In-service training should be directed to change the attitudes of nurses toward practice and learning."

- "In-service training should be introduced to mitigate the negative attitudes of the nurses."

- "Getting use to do it. Encourage nurses to attend."

- "They should go with changes. Adapt to changes and have open-minded attitudes, for example to ask yourself why do you do BP; assessing the wound. You can do a lot of things if you have the brain."

- "All registered nurses need to be updated to apply reflective practice in their practice."

- "Emphasise reflective practice in basic training of nurses."

- "Set reflective practice as a required standard for the practice of nursing to improve the standard of nursing care."

- "In-service training to be emphasized to update nurses."

- "Clinical nursing managers to demand the application of reflective practice by nurses and by them (managers) to observe bedside practice and improve it."

- "Nurses should be open to share their expertise with others and see how it can improve the community."

- "Proper bedside nursing should be revived."

- "Nurses should be encouraged and motivated by telling them that they have various gifts whereby they can provide care to the patients."

- "There is a need for consistency in supervision of nurses in the wards by the supervisors in order to make sure that nurses are applying reflective practice or practice quality care."

- "The enrolled nurses should also be included in this programme because they are always at patients' bedsides."

- "The nurse as an advocate for the patient has to reflect on patients' progress when giving a feedback to others."

- "Nurses should also apply the reflective practice in their patient-nurse relationship and in health education to the patients for example interpreting patients' diagnoses, treatment and their side effects."

- "In-service training of all nurses in reflective practice is important and necessary."

- "Reducing of the bureaucratic approach that inhibits improvement of quality of care because bureaucracy can inhibit the clinical manager to initiate the improvement of care."

- "Discussions should be held between stakeholders and Ministry of Health and Social Services (MOHSS) for example training institutions about the improvement of care at state hospitals."

- "The mind-set of registered nurses should be changed. Move from the thoughts of not regarding this, as not important."

- "We need to be trained and be positive and apply and evaluate ourselves."

- "Those who attended the training workshop should share in formation."

- "More workshops are needed for each and every nurse to be trained."

- "Involve all the nurses even the doctors so that all of us should have knowledge on reflective practice."

- "Nurses need to be educated not to do practice as routine but to change people's minds."

- "I did not notice any changes."

Summary of findings on the above question or item

The respondents revealed that some changes need to be made regarding the application of reflective practice programme, such as teaching all health providers not to do things simply as a routine, but to improve on what they used to do. It was also mentioned that even the doctors should have knowledge of reflective practice.

A request was made for increasing the number of workshops so that each and every nurse can be trained, and those who attended the workshops should share the information with colleagues. It was also mentioned that since the nurses are the advocating agents for the patients they have to reflect on patients' progress when giving feedback to others. Nurses should also apply reflective practice in their patient-nurse relationships and in health education to the patients for example to interpret patients' diagnoses, treatment and their side effects. The point was also made that inservice training in reflective practice for all nurses is important and needed.

There is a need for reduction of a bureaucratic approach that inhibits improvement of quality of care, in order to initiate and enhance the improvement of care. Continuous discussions between all stakeholders and the Ministry of Health and Social Services should be encouraged regarding the improvement of care in state hospitals. There is a need to have reflective practice as a required standard for the practice of nursing to improve the standard of nursing care. Clinical nursing managers should demand the application of reflective practice by nurses and by themselves to observe and improve bedside practice.

The atmosphere during the feedback session, the findings of which have been summarized above, was relaxed. The participants were open to tell their experiences and to share their views with others. The above comments of the participants correspond with what has been stated by Cirocco (2007), who indicated that important domains for examining proficiency in nursing practice include: effective management of rapidly changing situations, monitoring and ensuring the quality of healthcare practices.

\section{Conclusion}

This article delved into the evaluation of the reflective practice programme. It is assumed that reflective practice as the preparedness of registered nurses to think critically and to engage in reflection upon professional practice activities leads to the improvement of their performance.

The registered nurses who participated in the training and evaluation of the programme were deeply committed to the discussions. They have been motivated and were aware of their own responsibility for the improvement of their practice with regard to the application of reflective practice. The evaluation of the programme on reflective practice was dealt with through focus group discussion and face-to-face interviews. The participants were open to tell their experiences and to share their views with others. A request was made for increasing the number of workshops so that each and every nurse can be trained, and those who attended the workshops should share the information with colleagues. Some of the registered nurses expressed that what they like about this programme is the fact that it is a very good approach that keeps the nurses alert and reminds them to look back to the patients' conditions as well as to review their work and see where they can improve or where they need to teach others to improve.

\section{Acknowledgement}

This article was written and extracted from the study conducted by the author as "An educational programme to facilitate reflective practice of registered nurses in training hospitals in Windhoek with the permission of the Ethical and Research Committee of the Ministry of Health and Social Services in Namibia. Special thanks are extended to all the registered nurses who participated in this evaluation process of reflective practice programme for making themselves available in spirit of sharing their experiences and perceptions on the implementation of the programme which leads to the improvement of provision of nursing care to the patients.

\section{References}

[1] Bengtsson, M. \& Ohlsson, B. (2010). The Nursing and Medical students' motivation to attain knowledge. Nurse Education Today, 30(2), 150-156. http://dx.doi.org/10.1016/j.nedt.2009.07.005. 
[2] Cirocco, M. (2007). How reflective practice improves nurses' critical thinking ability. Gastroenterology Nursing, 30(6), 405-413. http://dx.doi.org/10.1097/01.SGA.0000305221.78403.e9.

[3] Forneris, S. G. \& Peden-McAlpine, C. (2006). Contextual Learning: A reflective learning intervention for nursing education. International Journal of Nursing Education Scholarship, 3(1, article 17), 1-18. http://dx.doi.org/10.2202/1548-923x.1254.

[4] Musal, B., Taskiran, C. Gursel, Y. Ozan, S. Timbil, S. \& Velipasaoglu, S. (2008). An example of programme evaluation: Project in undergraduate Medical education. Education for Health, 21(1), 113. Retrieved on 14 May, 2012 from http://www.educationforhealth.net.

[5] Wong, L.P. (2008). Focus group discussion: a tool for health and medical research. Singapore Med. Journal, 49(3), 256-260. 